\title{
EEG/EMG based Architecture for the Early Detection of Slip-induced Lack of Balance
}

\author{
Giovanni Mezzina ${ }^{1}$, Federica Aprigliano ${ }^{2}$, Silvestro Micera $^{2}$, Vito Monaco ${ }^{2}$, Daniela De Venuto ${ }^{1}$ \\ ${ }^{1}$ Dept.. of Electrical and Information Engineering, Politecnico di Bari \\ Via E.Orabona 4, 70125 Bari, Italy \\ ${ }^{2}$ The BioRobotics Institute, Scuola Superiore Sant'Anna \\ Viale Rinaldo Piaggio 34, 56025 Pontedera (PI), Italy
}

Email: \{giovanni.mezzina, daniela.devenuto\}@poliba.it, \{f.aprigliano, silvestro.micera, vito.monaco\}@santannapisa.it

\begin{abstract}
In this paper, we propose the preliminary version of a novel pre-impact fall detection (PIFD) strategy, optimized for the early recognition of balance loss during the steady walking.

The technique has been implemented in a multi-sensor architecture aiming to jointly analyzes the muscular and cortical activity. The physiological signals were acquired from 10 electromyography (EMG) electrodes on the lower limbs and 13 electroencephalography (EEG) sites all along the scalp.

Data from the EMGs are statistically treated and used both to identify abnormal muscular activities and to trigger the cortical activity assessment. The EEG computation branch evaluate the rate of variation of the EEG power spectrum density, named $\mathbf{m}$, to describe the cortical responsiveness in five bands of interest. Then, a logical conditions network allows the system to recognize the loss of balance induced by the slippage, by considering both the evaluated muscular parameters and the cortical ones.

Experimental validation on six adults (supported by the motion capture system) showed that the system reacts in a time compliant with the fall dynamic request $(403.16 \mathrm{~ms})$, ensuring a competitive detection accuracy (Sensitivity $=93.33 \%$, Specificity $=99.82 \%)$.
\end{abstract}

Keywords-Pre-impact Fall Detection, EEG, EMG, Kinematic

\section{INTRODUCTION}

In its annual report (last update Jan. 16, 2018 [1]) on the most common injury and death causes, the World Health Organization (WHO) reports the falls as the second leading cause of accidental or unintentional injury deaths worldwide [1]. Globally, about 646000 individuals (estimated value) die from falls. Approximately $28-35 \%$ of adults older than $70+$ years of age experiences up to 5-7 fall events per year, because it has been widely proved that the natural aging process alters the ability to address unexpected perturbations of balance, increasing the probability of falling $[1,2]$.

For these reasons, several research groups are still developing increasingly efficient fall prevention strategies to mitigate the harm of falls. In this context, the fall detection (FD) field aims to automatically detect the occurrence of a loss of balance, enabling proper on-demand fall protection systems (e.g., wearable airbags for hip protection, exoskeletons, etc.) $[3,4]$. A general classification of the FD strategies divides the proposed solutions in post-fall mobility detection and preimpact detection. The former type (i.e. post-fall mobility

This work was supported by the the project AMICO (Assistenza Medicale In COntextual awareness, AMICO_Project_ARS01_00900) by National Programs (PON) of the Italian Ministry of Education, Universities and Research (MIUR): Decree n.267. It was also supported by EU Commission through the H2020 project CYBERLEGs Plus Plus (The CYBERnetic LowEr-Limb CoGnitive Ortho-prosthesis Plus Plus, Grant Agreement no. 731931), the Italian National Institute for Insurance against accidents at work (INAIL) within the MOTU project (PPR-AI 1-2). detection) has the role of avoiding the "long lie" (i.e., the time spent on the floor following a fall), but it does not prevent from the fall-related injuries [5].

The pre-impact fall detection (PIFD) strategies can be generally defined as techniques that allow falls to be detected before the body-to-ground impact [5]. Specifically, they are designed to detect and eventually intervene (e.g., by activating on-demand protection system) in the early phase of the balance loss [6].

The performance of a PIFD strategy can be expressed in terms of accuracy - by considering the sensitivity and specificity parameters - and efficiency, evaluating the detection and the lead time [6].

To ease of reading, in this section we will often refer to a lack of balance (LoB) event by naming it "fall".

Commonly, the sensitivity parameter is defined as in eq.(1):

$$
\text { Se }(\%)=\left(\#(\operatorname{Tr} F) / N_{\text {fall }}\right) \cdot 100
$$

where \# $(\operatorname{Tr} F)$ is the number of correctly detected fall events and $N_{\text {fall }}$ is the total number of evaluated falls.

The specificity can be written as:

$$
S p(\%)=\left(\#(\operatorname{TrNF}) / N_{N \text { fall }}\right) \cdot 100
$$

where \# $(\operatorname{Tr} N F)$ is the amount of successfully detected nonfall activities, while $N_{\text {fall }}$ is the total number of the evaluated non-falls.

The PIFD strategy efficiency is evaluated in terms of lead time and detection time. The first one (i.e., the lead time) is defined as the time span between the fall detection and the bodyground impact. It takes into the account the time to actuate protection or compensatory measures.

In a complementary way, the detection time is the time range from the perturbation initiation and the fall detection.

According to [7] the time interval between the heel-strike and the fall impact can be estimated to be, on average, $0.9 \mathrm{~s}$ (range: $0.7-1 \mathrm{~s})$.

Table I summarizes some selected state of the art PIFD solutions $[4,6,8,9]$ by analyzing: the detection equipment, the lack of balance indicators, the implemented algorithm, the accuracy and efficiency of the used strategy. All the selected works concern the early LoB recognition in slip-induced falls, standardizing the comparison with our work.

Most of the PIFD solutions proposed by the literature base the fall detection on motion capture systems (MCSs) and kinematic fall indicators obtained by reflective markers on anatomic landmarks of the human body. Cameras mounted in fixed locations trace the trajectories of these markers $[6,8,9]$. Although these approaches ensure high accuracies $(\mathrm{Se}=88.5 \%$ - $100 \%$ and $\mathrm{Sp}=92.9 \%-99.2 \%$ ) and efficiency (detection time from 300 to $710 \mathrm{~ms}$ ), they are practically impossible to be 
TABLE I: PIFD STRATEGIES: STATE OF THE ART

\begin{tabular}{|c|c|c|c|c|c|}
\hline Spec. & [8] & [9] & [6] & [4] & Our work \\
\hline Technology & $\mathrm{MCS}+\mathrm{IMU}$ & MCS & MCS & $\begin{array}{l}\text { Hip Encoders } \\
\text { Ver: MCS }\end{array}$ & $\begin{array}{l}\text { EEG/EMG } \\
V e r: \text { MCS }\end{array}$ \\
\hline Indicator & $\begin{array}{l}\text { Trunk Angular } \\
\text { velocity }\end{array}$ & $\begin{array}{c}\text { Linear accel. of body } \\
\text { segments }\end{array}$ & $\begin{array}{l}\text { Head vertical acc., } \\
\text { upper arm and trunk } \\
\text { vertical vel., shank } \\
\text { frontal velocity, head } \\
\text { frontal angular } \\
\text { velocity }\end{array}$ & $\begin{array}{c}\text { MOS and COM } \\
\text { Hip angle }\end{array}$ & $\begin{array}{l}\text { Lower limbs } \\
\text { muscular activity }+ \\
\text { Reactive cortical } \\
\text { response to the LoB }\end{array}$ \\
\hline Algorithm & $\begin{array}{l}\text { Individualized } \\
\text { Threshold on velocity }\end{array}$ & $\begin{array}{l}\text { ICA+ Neural network } \\
\text { to classify walking vs } \\
\text { fall }\end{array}$ & $\begin{array}{l}\text { ARIMA model + } \\
\text { statistical based } \\
\text { thresholds }\end{array}$ & $\begin{array}{c}\text { Error function } \\
\text { between the hip } \\
\text { angles of the robot } \\
\text { with those predicted } \\
\text { by an adaptive } \\
\text { oscillators }\end{array}$ & $\begin{array}{c}\text { Muscular score AND } \\
\text { (logical) Cortical } \\
\text { Dynamics Change } \\
\text { Detector }\end{array}$ \\
\hline Accuracy & $\begin{array}{c}\mathrm{Se}=100 \% \\
\mathrm{Sp}=96.5 \%\end{array}$ & $\begin{array}{l}\mathrm{Se}=92.7 \% \\
\mathrm{Sp}=98 \%\end{array}$ & $\begin{array}{l}\mathrm{Se}=88.5-94.7 \% \\
\mathrm{Sp}=92.9-99.2 \%\end{array}$ & Not specified & $\begin{array}{l}\mathrm{Se}=93.33 \% \\
\mathrm{Sp}=99.82 \% \\
\end{array}$ \\
\hline$\underset{* 1}{\text { Detection time (ms) }}$ & 300 & 351 & $620-710$ & $\sim 350$ & 403 \\
\hline $\begin{array}{c}\text { Protection System } \\
\text { Activation }\end{array}$ & $x$ & $x$ & $x$ & $\begin{array}{c}\checkmark \\
\text { Active Pelvis Orthosis }\end{array}$ & $x$ \\
\hline Dataset & $10 \mathrm{OA}$ & $15 \mathrm{YA}$ & $60 \mathrm{YA}$ & $8 \mathrm{OA}$ and $2 \mathrm{TA}$ & $6 \mathrm{YA}$ \\
\hline
\end{tabular}

implemented in real life applications [7]. Moreover, all the proposed solutions (except [4]) do not activate external protective devices even if they ensure a detection time that paves to the possibility of effectively trigger them. The authors in [4] analyze the error function between the hip angles of the robot (exoskeleton) with those predicted by adaptive oscillators. If a $\mathrm{LoB}$ is detected, counteracting torques are supplied by the Active Pelvis Orthosis at the hip joints to promote the balance recovery.

In this preliminary work, we investigated a new PIFD strategy for the early detection of a lack of balance (LoB) when unexpected slippages delivered during steady walking.

More in details, the proposed technique was implemented with a multi-sensor architecture that jointly analyzes the muscular activity and the cortical involvement of a subject that actively reacts to recover the perturbed balance. Specifically, the muscular activity was acquired by using surface electromyography (EMG), while the cortical dynamics was investigated via electroencephalography (EEG).

The proposed architecture is based on statistic-derived thresholds and a network of logical conditions, which are progressively individualized during the system use. It allows the approach to require very limited personalization.

Experimental validation, via MCS, showed that the system reacts in a time compliant with the fall dynamic request (403.16 ms), ensuring a high detection accuracy $(\mathrm{Se}=93.33 \%$, $\mathrm{Sp}=99.82 \%$ ).

The paper is structured as follows: Sec. II outlines the detection algorithm, also providing details about the experimental protocol and setup. Sec. III shows some preliminary experimental results and Sec. IV provides the conclusions.

\section{THE PIFD SYSTEM}

A general block diagram of the proposed multi-sensor architecture is shown in Fig.1. According to the figure, during the experimental trials, the subjects were asked to manage unexpected slippages occurred during steadily walking at their preferred speed (Perturbation Protocol Fig.1). Data from EEG and EMG electrodes are wirelessly collected by a common gateway and treated by a novel detection algorithm, which aims to extract useful information about the cortical dynamics in the reactive balance recovery. In general, the implemented algorithm is composed of two main units: the EMG computation branch (EMG Side - Fig.1) and the EEG ones (EEG Side - Fig.1).

The first one (i.e., EMG computation branch) realizes a trigger for the EEG signals analysis. In this way, the proposed algorithm focuses the EEG analysis only in correspondence of a specific gait phase. It permits to exclude from the computation the cerebral activity that is not strictly related to the specific movement.

Firstly, the EMG signals are properly treated to identify the contraction onsets of each monitored muscle. This procedure, named Trigger Extraction in Fig.1, translates a double precision EMG signal in a binary waveform.

All the contraction onsets can be used to trigger the EEG computation, however only one muscle (bilaterally) was selected to manage the cortical branch working. In this application, the Gastrocnemii (left and right) were selected as Master Triggers (MTs).

The MT enables the EEG signals analysis at every step (both the sides, right and left), allowing the system to investigate the cortical activity related to both unperturbed steps and compensatory ones.

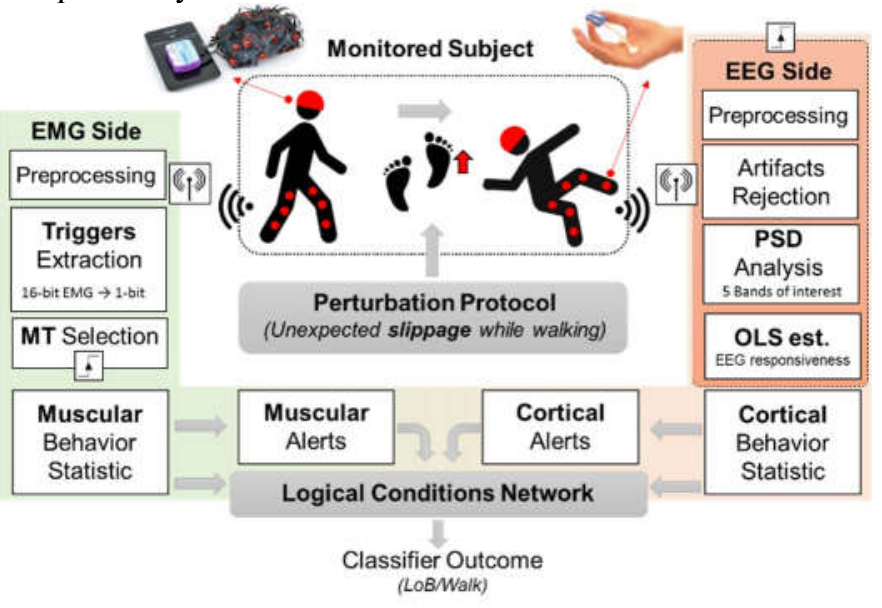

Fig.1 Overview of the proposed multi-sensor architecture 
Specifically, the EEG side aims to evaluate the variation of the EEG power spectrum density, quantifying the cortical responsiveness in five bands of interest (OLS est. - Fig.1). The evaluated EMG and EEG parameters are then used to build a progressive statistic of the general muscular and cortical behaviors near the MTs onset. The extracted statistics are used to train a logical conditions network. It permitted identifying abnormal behaviors in the muscle contractions and the reactive response in the cortical dynamics when the subject experienced a gait cycle perturbation. The network concludes the operative flow, returning the final classification (walking or balance loss).

\section{A. Perturbation Protocol}

The perturbations was provided by a custom-made split-belts treadmill named SENLY [10] and consisted of sudden forward movements toward the antero-posterior direction. Specifically, the selected belt was accelerated and decelerated to $0 \mathrm{~m} / \mathrm{s}$ with a triangular speed profile (slope $8 \mathrm{~m} / \mathrm{s}^{2}$ and total displacement of $0.15 \mathrm{~m}$ ).

The belt movement was triggered by detecting the heel strike of the foot appointed for the perturbation.

After a first acclimation stage $(\sim 5 \mathrm{~min})$, the protocol consisted of a series of 10 consecutive trials in which the subject gait was perturbed by a slippage. The slippages were equally delivered alternating right foot related belt and the left foot one. In this respect, a demonstrative sequence of frames, concerning the implemented experimental protocol, is shown in Fig. 2.

\section{B. Experimental Setup}

The PIFD strategy is based on a multi-sensor acquisition system consisting of a 32-channel wireless EEG headset, 10 wireless EMG surface electrodes and 23 reflective markers for the $3 \mathrm{D}$ kinematics reconstruction. All the devices were worn by the subjects as in Fig.2.

Specifically, 10 surface EMG channels were monitored from (bilateral muscle groups): Anterior Tibialis, Lateral Gastrocnemius, Vastus Medialis, Rectus Femoris, and Biceps Femoris. The EMG signals were sampled at $500 \mathrm{~Hz}$ with 16bit resolution.

Contextually, 13 EEG sites were monitored: F3, Fz, F4, C3, $\mathrm{Cz}, \mathrm{C} 4, \mathrm{Cp} 5, \mathrm{Cp} 1 \mathrm{Cp} 2, \mathrm{Cp} 6, \mathrm{P} 3, \mathrm{Pz}, \mathrm{P} 4$ [11, 12], AFz as ground and the $\mathrm{A} 2$ (right ear-lobe) as the reference electrode. The EEG data were sampled at $500 \mathrm{~Hz}$ with 24-bit resolution. Both EEG and EMG were transmitted via Bluetooth Low Energy protocol (BLE) and synchronized by a dedicated gateway.
To temporally validate the proposed PIFD strategy, data collected were analyzed offline by using MATLAB ${ }^{\circledR} 2017 \mathrm{~b}$ and the 3D trajectory of the markers on the subject's lower limbs was recorded by an 8-camera MCS.

The markers were mounted on: anterior superior iliac spines, sacrum, prominence of the greater trochanters external surface, lateral and medial epicondyle of the femurs, heads of fibula, lateral and medial malleolus, calcaneus, $1^{\text {st }}$ and $5^{\text {th }}$ metatarsal heads, midfemurs and midshaft of the tibia. Data from the MCS were sampled at $100 \mathrm{~Hz}$.

\section{Data Pre-Processing}

Data from EEG and EMG equipment were collected from a gateway and stored in a MATLAB ${ }^{\circledR}$ workspace for further analysis. Kinematic data and electrophysiological signals underwent a pre-processing stage, which is summarized in Table II in terms of allowed frequency span and used filter type. Both EEG and EMG operate the filtering stage before the transmission (labeled as Online in Table II). Concerning the kinematic record, the missing data were estimated by using cubic spline interpolation.

\section{The EMG Computation Branch}

\section{1) Trigger Extraction}

The first step of the detection algorithm was the muscle triggers extraction [13]. It consisted of a dynamic threshold approach, in which each EMG signal (16-bit) was converted in a binary signal (named trigger). It is high when the muscle is contracted, low otherwise. Fig. 3 shows all the steps for the trigger generation. The method, widely treated in $[14,15]$, consists of comparing the average signal power on a time span of $\mathrm{M}=500 \mathrm{~ms}(\mathrm{PM}-$ Fig. 3) and the average signal power on a time span of $\mathrm{N}=250 \mathrm{~ms}$ (i.e., the last $250 \mathrm{~ms}$ of the $\mathrm{M}$ register, PN - Fig.3). The process was refreshed sample-bysample. For the $\mathrm{i}^{\text {th }}$ sample, PN was compared with the PM. If $\mathrm{PN}$ is higher than PM, the trigger goes high, otherwise zero. The first panel of the Fig. 3 shows, sample-by-sample, the PM and $\mathrm{PN}$ values, while the second panel demonstrates how the trigger fits the raw EMG [15].

For ease of reading, only one onset of the MT has been

\begin{tabular}{|c|c|c|c|c|}
\hline Signal & $\begin{array}{l}\text { Bandpass } \\
\text { Freq. span }\end{array}$ & Filter & Type & Notch* \\
\hline EMG & $10 \mathrm{~Hz}-240 \mathrm{~Hz}$ & $\begin{array}{c}8^{\text {th }} \text { ord. } \\
\text { Butterworth }\end{array}$ & Online & $\checkmark$ \\
\hline EEG & $1 \mathrm{~Hz}-40 \mathrm{~Hz}$ & $\begin{array}{c}8^{\text {th }} \text { ord. } \\
\text { Butterworth }\end{array}$ & Online & $\checkmark$ \\
\hline Kinematic & $<10 \mathrm{~Hz}$ & $\begin{array}{c}\text { Zero-lag } \\
4^{\text {th }} \text { ord. } \\
\text { Butterworth }\end{array}$ & Offline & $x$ \\
\hline
\end{tabular}

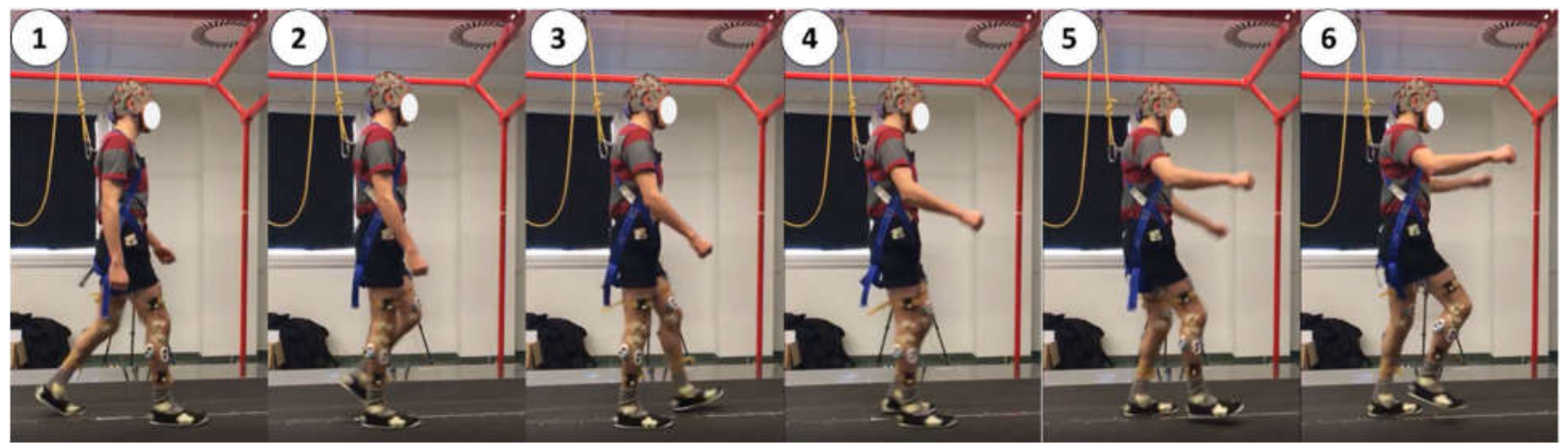

Fig.2 Chronologically ordered demonstrative frames (6 frames) that shows the implemented experimental protocol. All the participants were secured by a safety harness attached to an overhead track. 

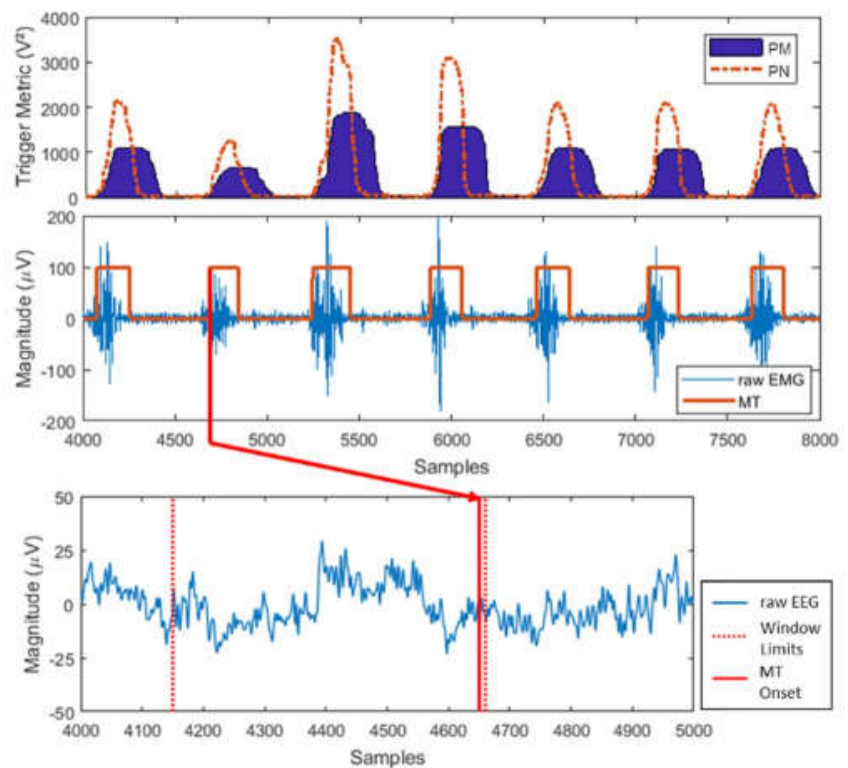

Fig.3 Trigger extraction procedure: (a) PM and PN values to be compared; (b) MT overlapped to the raw EMG signal; (c) the MT onset enables an EEG window extraction (time span between the two dashed red lines).

considered as the EEG computing branch trigger in the Fig. 3 (red arrow).

2) Muscular Activity Pattern and Score Assignement The extracted triggers (i.e., 10 binary signals) were transmitted to a computation block that assigns, in correspondence of every MT onset, a statistics coherence coefficient between the step to be evaluated and the general muscles behavior during the steady walking.

The assignment procedure is based on the hypothesis of a moderate inter-step repeatability when the subject is involved in an unperturbed walking. Differently, in the presence of a perturbation, due to the parallel activation of normally nonconcurrent muscles (e.g., co-contraction of agonist and antagonist muscles [11]), it was expected an abnormal behavior.

In this respect, it has been developed a routine, named Query, which interrogates the state of each evaluated trigger, starting from the MT onset for a time interval of $20 \mathrm{~ms}$ (i.e., 11 samples).

For each trigger (or muscle), the final value is then provided according to a predominance-based approach. The resulting binary vector, which considers the states of all the muscles, is named Muscular Activity Pattern (MAP), MAP $\in \mathbb{R}^{\operatorname{Tr}}$ with $\mathrm{Tr}=10$ the number of evaluated triggers.

For sake of clarity, considering the $\mathrm{j}^{\mathrm{th}}$ muscle, if the number of " 1 " in the evaluated window (i.e., 11 samples) is $>\operatorname{Tr} / 2$ (i.e., $5)$, the resulting MAP[j] value is set to " 1 ", " 0 " otherwise.

To extract the statistically "most probable" muscular pattern, it was introduced proper weights based on the statistical occurrence. The procedure is composed of two steps:

1. The system studies the occurrence of a specific logic state (e.g., "1"), for each single trigger in correspondence of the MT onset (e.g., the Right Lateral Gastro.: R_LG). This computation is done on a selected statistic sample (Analyzed Contractions - Fig.3). This process leads to the definition of a vector, $\mathbf{F}_{1 \mathbf{R}} \in \mathbb{R}^{\mathrm{Tr}}$, with " 1 " the selected state, "R" the reference to the considered MT. The generic element of $\mathbf{F}_{1 \mathbf{R}}$ can be defined as:

$$
f_{1 R, T r_{i}}=\left.\frac{\#\left(T r_{i}==1\right)}{N_{o b s}}\right|_{M T=R_{-} L G}
$$

where $\#\left(\operatorname{Tr}_{i}==1\right)$ represents the number of " 1 " collected, by the $\mathrm{i}^{\mathrm{th}}$ trigger, during the trial and $\mathrm{N}_{\mathrm{obs}}$ is the number of observations that compose the statistic sample. The eq. (3) ensures that $f_{1 R, T r_{i}} \in[0,1]$. Contextually, the architecture extracts the $\mathbf{F}_{\mathbf{0 R}}=1-\mathbf{F}_{1 \mathbf{R}}$.

2. In a similar manner, the system extracts the $\mathbf{F}_{1 \mathrm{~L}} \in \mathbb{R}^{\mathrm{Tr}}$ and the $\mathbf{F}_{0 \mathrm{~L}} \in \mathbb{R}^{\mathrm{Tr}}$, by considering as MT the left lateral Gastro. (L_LG).

The Fig. 4 shows an experimental extraction of the triggers evolution during a trial, by considering R LG as MT. The label "Analyzed Contractions" refers to the observations that compose the statistic sample for the $\mathbf{F}_{1 \mathrm{R}}$ and $\mathbf{F}_{\mathbf{0 R}}$ definition. The label "Steady Walking" refers to the R_LG contractions during the unperturbed walking, while the "Perturbation \& Recovery" concerns the perturbed steps and the compensatory ones. Fig. 4 also shows an experimentally derived numeric example of the weights vectors $\mathbf{F}_{1 \mathbf{R}}, \mathbf{F}_{\mathbf{0}}$.

Once the weights vectors are defined (i.e., $\mathbf{F}_{1 \mathrm{R}}, \mathbf{F}_{\mathbf{0 R}}, \mathbf{F}_{1 \mathrm{~L}}, \mathbf{F}_{\mathbf{0 L}}$ ) the Query routine is applied at every MT contraction, generating a step-by-step MAP.

In this respect, the Fig. 5.a sketches the processing algorithm for the statistical coherence degree assessment of the $i^{\text {th }}$ step related MAP.

The MAP triggered by the R_LG (R_MAP in Fig. 4.a) is

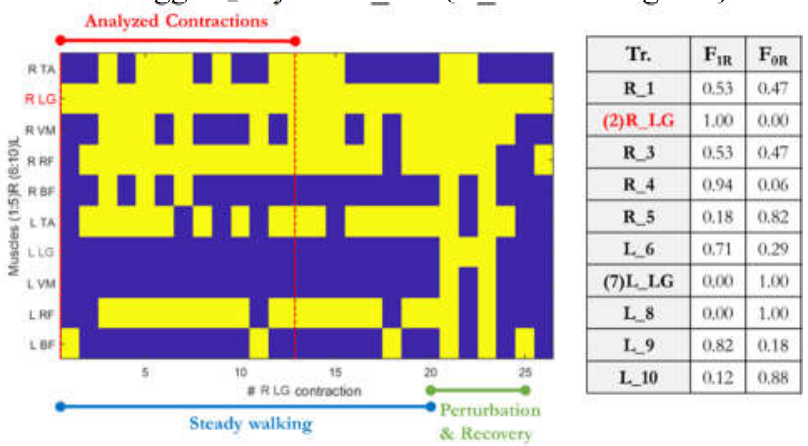

Fig.4 Muscular triggers behavior near the R_LG activation. Each row represents a muscle trigger, the yellow square means " 1 ", while the blue ones " 0 ". The table shows the weights related to $\mathbf{F}_{1 \mathrm{R}}$ and $\mathbf{F}_{\mathbf{0 R}}$

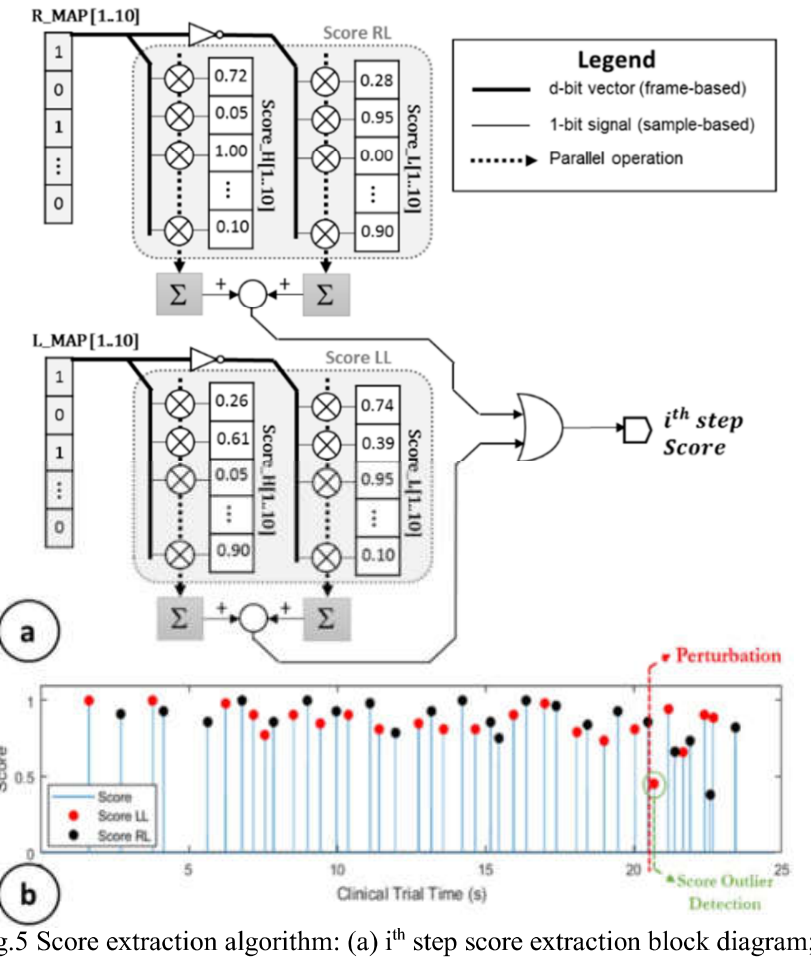

(b) overall score trend (experimental data from Sub.6 - Trial 4) 
multiplied, element-by-element, by the $\mathbf{F}_{1 \mathbf{R}}$, while the MAP negative logic copy is multiplied by $\mathbf{F}_{\mathbf{0}}$.

Considering the R LG contraction, the products are then progressively summed, to define the specific score according to eq. (4):

$$
\text { Score } R L=\frac{\sum_{i=1}^{T r} M A P(i) * F_{1 R}(i)+\sum_{i=1}^{T r} \overline{M A P(l)} * F_{0 R}(i)}{\sum_{i=1}^{T r} \max \left(\left[F_{1 R}(i), F_{0 R}(i)\right]\right)}
$$

where the denominator allows the system to normalize the score (i.e. ScoreRL. $\in[0,1]$ ). The same procedure is applied to the MAPs derived by using L_LG as MT (i.e. L_MAP - Fig. 5.a). Finally, the Fig.5.b shows an experimentally extracted example of the overall score trend (Score RL U Score LL) across a clinical trial.

\section{E. The EEG Computation Branch}

\section{1) Online Artifacts Rejection}

The detection of the lateral Gastrocnemius (both sides) rising edge, enables the EEG processing branch (see Fig. 3).

Specifically, a 1.04 s (520 samples) long EEG time window was extracted across the MT as $1 \mathrm{~s}$ (500 samples) before and $0.04 \mathrm{~s}$ (20 samples) after the trigger. This EEG chunk underwent an on-line Riemannian Artifact Subspace Reconstruction (rASR) [16], which is an online artifacts rejection approach for mobile EEG application.

\section{2) Cortical Responses Analysis}

The authors in [12] investigated the cortical dynamics while subjects experienced perturbations, even unexpected, which could critically affect their orthostatic quite stance [12]. They recorded a first increase of the power spectral density (PSD) in the range $4-13 \mathrm{~Hz}$ ( $\vartheta$ and $\alpha$ waves), typically related to sensorimotor and cognitive functions (e.g., fall perception phase) $[12,6]$. In addition, the authors also show an increase of energy of the PSD in the range $13-40 \mathrm{~Hz}$ ( $\beta$ I, II, III bands). The $\beta$ bands are typically involved in motor functions [4]. The sharp changes in the cortical activity typically reached their peaks in about 80-171 ms after stimulus [12].

Basing on these evidences, in our previous /work [16], we widely investigated changes in the cortical involvement when subjects were actively managing unexpected slippages delivered during steady walking. We also demonstrated, through experimental results, that the proposed algorithm is able to distinguish between steady walking and early reactive balance recovery [16].

For this reason, the EEG computation branch of the proposed architecture exploits the algorithm behind the clinical study in [16] to manage the EEG side of the PIFD strategy.

The Fig. 6 shows the implemented procedure for the cortical dynamics assessment.

Briefly, the artifacts-free EEG block is split in 10 overlapped 500-samples long time windows, running along the whole chunk (with 2 samples, or equivalently $4 \mathrm{~ms}$, step). For each of them, the system computes the FFT and extract the energy of the PSD in 5 bands of interests (BoI): $\vartheta(4-7 \mathrm{~Hz}), \alpha(8-12 \mathrm{~Hz})$, $\beta$ I, $\beta$ II, $\beta$ III rhythms $(13-15,15-20,18-28 \mathrm{~Hz})$. The sums on a specific BoI are represented in Fig. 5 by the labels: $\Sigma_{1 \ldots 10}$. For each BoI, a linear model of the measured data (i.e., $\Sigma_{1 \ldots 10}$ ) is extracted via ordinary least squares (OLS - Fig.6) fitting. The slope of this model, $m$ (i.e, $\hat{\mathrm{p}}(1)$ ), is then used to describe the cortical responsiveness [16]. According to the literature [12], a sharp increment of $m$ indicates a greater involvement of the cortical area.
The Fig. 6 shows the EEG computation branch for a single EEG channel (ith EEG Ch - Fig. 6) and a specific BoI (yellow area - Fig.6). The structure in Fig.6 is repeated for all the thirteen EEG channels and considering the five BoIs. It leads to a total of $65 \mathrm{~m}$ values per MT activation (i.e., 13 channels * $5 \mathrm{BoI})$.

\section{F. Logical Conditions Network}

As above-stated the proposed multi-sensor architecture jointly analyzes the muscular activity and the cortical involvement of a subject that actively reacts to recover the perturbed balance. For this purpose, the system statistically derives thresholds and a network of logical conditions to detect, with proper accuracy, the loss of balance induced by the slippage.

More in details, the threshold extraction procedure consists of two steps:

1. The system considers an initial observation window. In this preliminary work, the selected initial window coincides with the "Analyzed Contractions" area in Fig. 3. From this time window, the system extracts:

- the $5^{\text {th }}$ percentile of the evaluated muscular scores (Sec. II.D.2);

- the $95^{\text {th }}$ percentile of the $m$ values, for each BoI, averaged on 4 functional groups of EEG channels:

- Supplementary Motor Area (SMA): $\{\mathrm{F} 3, \mathrm{Fz}$, F4\};

- Motor area (M1): $\{\mathrm{C} 3, \mathrm{Cz}, \mathrm{C} 4\}$;

- Sensory-motor area: $\{\mathrm{Cp} 5, \mathrm{Cp} 1, \mathrm{Cp} 2, \mathrm{Cp} 6\}$;

- Parietal area: $\{$ P3, Pz, P4 $\}$

2. Once the initial thresholds are extracted the system waits for a new MT contraction. If the MT rising edge is detected the muscular score is compared with the initial threshold (iThr). If $\mathrm{i}^{\text {th }}$ score $<$ iThr the muscular side activates an alert.

Contextually, if the $m$ values, in the functional group and for each BoI, overcome $(>)$ the initial dedicated thresholds (i.e., $95^{\text {th }}$ percentile), also the EEG side reacts by activating several alerts. An overall of 20 alerts can be generated by the EEG side ( 5 BoIs * 4 functional groups).

After the comparisons the thresholds are updated, taking into account the new values of score and $m$.

Finally, the architecture will enable a potential feedback procedure, if there is a parallel presence of a muscular alert and, at least, the $51 \%$ of the available EEG warnings.

\section{EXPERIMENTAL RESULTS}

Six healthy young subjects ( 5 males, 1 female, $26.3 \pm 2.4$ years, $64.5 \pm 9.8 \mathrm{~kg}, 1.71 \pm 0.06 \mathrm{~m}$ ) were involved in this study. Subjects walked at an average speed of $1.10 \pm 0.07 \mathrm{~m} / \mathrm{s}$ $\mathrm{m} / \mathrm{s}$ (range: $1-1.15 \mathrm{~m} / \mathrm{s}$ ). No falls were reported during the trials. All participants were able to recover their balance.

Before starting the experimental sessions, all participants signed an informed consent. Research procedures were in accordance with the Declaration of Helsinki and was approved by the Local Ethical Committee.

\section{A. Architecture Performance}

The performance of a PIFD strategy is quantified in terms of accuracy - by considering the sensitivity ( $\mathrm{Se}$ - eq (1)) and specificity ( $\mathrm{Sp}$ - eq (2)) parameters - and efficiency, evaluating the detection and lead time (DT and LT).

Table III summarizes these parameters, focusing on the intersubject variability. 


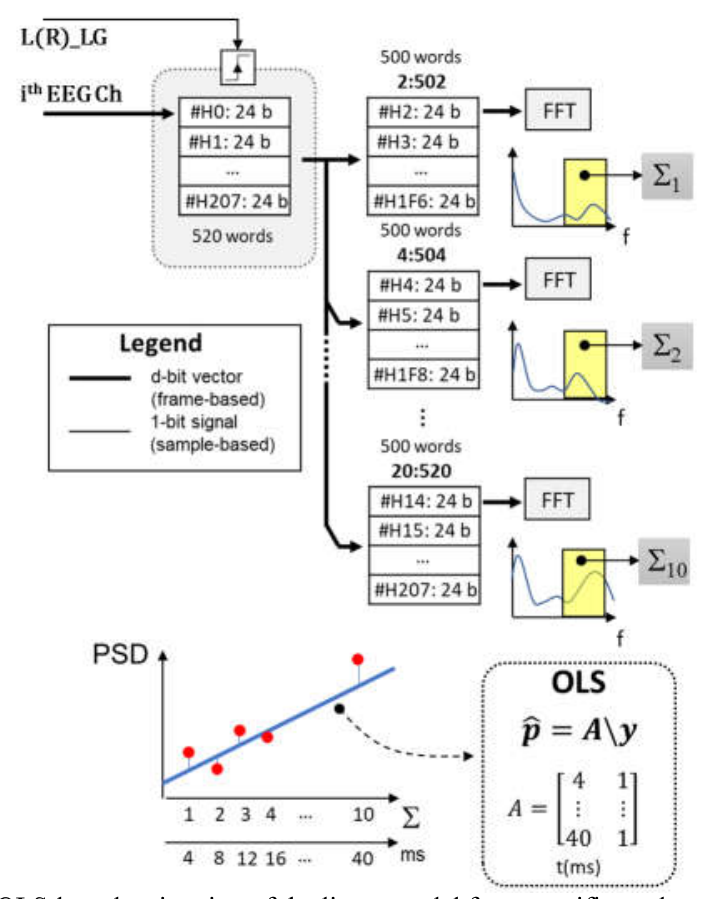

Fig.6 OLS-based estimation of the linear model for a specific analyzed BoI.

The proposed multi-sensor architecture shows a sensitivity of $93.33 \pm 5.16 \%$ and a specificity of $99.82 \pm 0.16 \%$. The table also reports the false alarms (FA) detected during the trials. On average, only $1 \pm 0.89 \mathrm{FA}$ was registered during each run (10 trials per subject).

The system detection time is, on average (on all the subject), $403.16 \pm 71.82 \mathrm{~ms}$. The LT showed in the table is computed considering the difference between $900 \mathrm{~ms}$ (i.e., time interval between the heel-strike and the fall impact) and the mean value of the DT. The LT represents a quantitative measure of the time available time to actuate protection or compensatory strategies.

\section{B. System Timing}

It is important to highlight that most of the DT is related to the MT activation. In fact, considering the MT rising edge as the "zero-time", the architecture asks for: $40 \mathrm{~ms}$ of EEG register filling, $2.23 \pm 0.54 \mathrm{~ms}$ for the FFT and BoI extraction, $0.32 \pm 0.07 \mathrm{~ms}$ to extract the $m$ values and $0.12 \pm 0.05 \mathrm{~ms}$ to realize the thresholds comparisons. Thus, in the worst case the processing chain requests only $43.33 \mathrm{~ms}$.

\section{CONCLUSIONS}

The paper described a step-by-step design of a multi-sensor architecture, implementing a preliminary version of an innovative PIFD strategy. The system was tested and optimized for the early detection of balance losses when unexpected slippages occur during the walking.

The architecture is composed of a wearable and wireless acquisition system: 10 surface EMGs and 13 EEGs electrodes, which sends data to a main PC. This latter analyzes the physiological data (EEG/EMG) extracting a network of statistic-based thresholds.

Experimental validation on six young adults demonstrated that the system recognize a lack of balance with a sensitivity of $93.33 \%$ and a specificity of $99.82 \%$. Finally, the system $r$ requests, on average, $403.16 \mathrm{~ms}$ to recognize the LoB, of which only $43.33 \mathrm{~ms}$ are due to the parameters extraction.
TABLE III: PIFD ACCURACY AND EFFICIENCY CHARACTERIZATION

\begin{tabular}{|c|c|c|c|c|c|}
\hline Sub & $\mathbf{S e}^{\boldsymbol{*}_{\mathbf{1}}}(\mathbf{\%})$ & $\mathbf{S p}(\mathbf{\%})$ & $\mathbf{F A}$ & $\begin{array}{c}\text { DT }[\boldsymbol{\mu} \pm \boldsymbol{\sigma}] \\
(\mathbf{m s})\end{array}$ & $\begin{array}{c}\mathbf{L T} \\
(\mathbf{m s})\end{array}$ \\
\hline $\mathbf{1}$ & 90 & 99.81 & 1 & $389.83 \pm 97.49$ & 510.17 \\
\hline $\mathbf{2}$ & 100 & 99.63 & 2 & $514.61 \pm 99.31$ & 385.39 \\
\hline $\mathbf{3}$ & 100 & 100 & 0 & $319.76 \pm 107.99$ & 580.24 \\
\hline $\mathbf{4}$ & 90 & 99.64 & 2 & $380.23 \pm 79.24$ & 519.77 \\
\hline $\mathbf{5}$ & 90 & 99.83 & 1 & $460.57 \pm 91.36$ & 439.43 \\
\hline $\mathbf{6}$ & 90 & 100 & 0 & $354.01 \pm 108.47$ & 545.99 \\
\hline${ }^{* 1} \mathrm{~N}_{\text {fall }}=10$ for all the subjects Se value.
\end{tabular}

The here-proposed PIFD strategy has been designed to be computationally suitable for the implementation on FPGA or microcontroller. The experimentally measured architecture performance (accuracy and detection time) paves the way to the system applicability in real time applications. Despite this, the identification of the proper protection or mitigation strategies (e.g., by using wearable robotic platforms) and the improvement of the acquisition system wearability is still under investigation.

\section{References}

[1] World Health Organization. "WHO global report on falls prevention in older age." Online Updated (January 16, 2018): https://www.who.int/news-room/fact-sheets/detail/falls

[2] Vellas B, Cayla F, Bocquet H, de Pemille F, Albarede JL. Prospective study of restriction of activity in old people after falls. Age Ageing. 1987;16:189-93.

[3] Tamura T, Yoshimura T, Sekine M, Uchida M, Tanaka O. A wearable airbag to prevent fall injuries. IEEE Trans Inf Technol Biomed. 2009;13:910-4.

[4] Monaco, V., et al. "An ecologically-controlled exoskeleton can improve balance recoyery after slippage." Scientific reports 7 (2017): 46721.

[5] Mubashir M, Shao L, Seed L. A survey on fall detection: principles and approaches. Neurocomputing. 2013;100:144-52.

[6] Hu, Xinyao, and Xingda Qu. "Pre-impact fall detection." Biomedical engineering online 15.1 (2016): 61.

[7] Beschorner, Kurt E., Mark S. Redfern, and Rakié Cham. "Earliest gait deviations during slips: implications for recovery." IIE Transactions on Occupational Ergonomics and Human Factors 1.1 (2013): 31-37.

[8] Liu J, Lockhart TE. Automatic individual calibration in fall detection: an integrative ambulatory measurement framework. Comput Methods Biomech Biomed Eng. 2013;16:1860-72

[9] Martelli D, at al. Pre-impact fall detection: optimal sensor positioning based on a machine learning paradigm. PLoS ONE. 2014;9(18):e92037.

[10] L. Bassi Luciani, et al. "Design and evaluation of a new mechatronic platform for assessment and prevention of fall risks," J NeuroengRehabil, vol. 9, p. 51, 2012

[11] M. de Tommaso, E. Vecchio, K. Ricci, A. Montemurno, D. De Venuto and V. F. Annese, "Combined EEG/EMG evaluation during a novel dual task paradigm for gait analysis," 2015 6th International Workshop on Advances in Sensors and Interfaces (IWASI), Gallipoli, 2015, pp. 181-186. doi: 10.1109/IWASI.2015.7184949

[12] Solis-Escalante, T. et al. "Cortical dynamics during preparation and execution of reactive balance responses with distinct postural demands." NeuroImage 188 (2019): 557-571.

[13] D. De Venuto, V. F. Annese, M. Ruta, E. Di Sciascio and A. L. Sangiovanni Vincentelli, "Designing a Cyber-Physical System for Fall Prevention by Cortico-Muscular Coupling Detection," in IEEE Design \& Test, vol. 33, no. 3, pp. 66-76, June 2016. doi: 10.1109/MDAT.2015.2480707

[14] V. F. Annese, M. Crepaldi, D. Demarchi and D. De Venuto, "A digital processor architecture for combined EEG/EMG falling risk prediction," 2016 Design, Automation \& Test in Europe Conference \& Exhibition (DATE), Dresden, 2016, pp. 714-719.

[15] V. F. Annese and D. De Venuto, "FPGA based architecture for fall-risk assessment during gait monitoring by synchronous EEG/EMG," 2015 6th International Workshop on Advances in Sensors and Interfaces (IWASI), Gallipoli, 2015, pp. 116-121. doi: 10.1109/IWASI.2015.7184953

[16] Mezzina, G., Aprigliano, F., Micera, S., Monaco, V., De Venuto, D. "Cortical reactive balance responses to unexpected slippages while walking: a pilot study". Proceedings of 41st International Engineering in Medicine and Biology Conference. unpublished 